\title{
De la communication à l'incommunication ?
}

In: Communication et langages. N¹46, 4ème trimestre 2005. pp. 3-18.

\section{Résumé}

En travaillant sur les « technologies de l'information et de la communication » (TIC), l'auteur en est venu à questionner une apparente évidence : la mobilisation de la notion de communication comme concept clé des Sciences de l'information et de la communication (SIC). Aussi, après un rapide retour critique sur les deux modèles canoniques du télégraphe et de l'orchestre, qu'il renvoie dos à dos, nous propose-t-il une exploration de la notion $A^{\prime}$ incommunication à travers la relecture de deux textes fort distincts, l'CEdipe de Sophocle dominé selon lui par la figure de « l'aveuglement communicationnel » et Solaris de Stanislas Lem, un classique de la science-fiction qui met en scène, selon Pascal Robert, le " pessimisme communicationnel ». L'auteur nous propose alors de situer la notion d' incommunication face au principe de Palo Alto selon lequel « on ne peut pas ne pas communiquer ", avant de conclure sur son indispensable conjuration.

Citer ce document / Cite this document :

Robert Pascal. De la communication à l'incommunication?. In: Communication et langages. N¹46, 4ème trimestre 2005. pp. 318.

doi : $10.3406 /$ colan.2005.3372

http://www.persee.fr/web/revues/home/prescript/article/colan_0336-1500_2005_num_146_1_3372 


\section{De la communication à l'incommunication?}

On a souvent dénoncé - et à juste titre - une attitude qui vise à penser la communication interpersonnelle à partir du "modèle télégraphique/technologique ». On s'interroge beaucoup moins sur la pertinence qu'il peut $y$ avoir à utiliser explicitement ou implicitement un modèle de la communication interpersonnelle, singulièrement le "modèle de l'orchestre " ${ }^{1}$, pour penser les technologies de l'information et de la communication (TIC). On en vient facilement, en effet, à la suite des travaux sur la réception et/ou de la sociologie de l'usage à accepter sur le mode de l'évidence que la communication interpersonnelle serait en quelque sorte le "bon " modèle, l'aune à laquelle il conviendrait de penser les TIC. C'est justement cette évidence que voudrait soumettre à la question cette réflexion sur l'incommunication.

\section{LE TÉLÉGRAPHE, L'ORCHESTRE ET L'INCOMMUNICATION}

Car il semblerait qu' "entrer dans l'orchestre " soit une opération sinon simple et totalement évidente, tout au moins relativement aisée à réaliser : globalement les hommes d'une société donnée rentrent dans l'orchestre sans trop de problèmes ni de questions et en supportent les contraintes. Ce n'est qu'à la marge que peuvent se dévoiler quelques cas d'insertion difficile. Si vous possédez le code, l'intégration est guère problématique : les difficultés surgissent au croisement, à l'articulation de deux codes - lorsque les uns ne possèdent pas le code de l'autre et croient que leur manière de faire, locales, sont universellement valables.

1. Cf. Yves Winkin (éd.), La nouvelle communication, Paris, Le Seuil, 1981 et Yves Winkin, Anthropologie de la communication, Paris, Le Seuil, 2001.
ÉPISTÉMOLOGIE

\section{PASCAL ROBERT}

En travaillant sur les « technologies de l'information et de la communication " (TIC), l'auteur en est venu à questionner une apparente évidence : la mobilisation de la notion de communication comme concept clé des Sciences de l'information et de la communication (SIC). Aussi, après un rapide retour critique sur les deux modèles canoniques du télégraphe et de l'orchestre, qu'il renvoie dos à dos, nous propose-t-il une exploration de la notion d'incommunication à travers la relecture de deux textes fort distincts, l'Edipe de Sophocle dominé selon lui par la figure de "l'aveuglement communicationnel " et Solaris de Stanislas Lem, un classique de la science-fiction qui met en scène, selon Pascal Robert, le "pessimisme communicationnel ". L'auteur nous propose alors de situer la notion d'incommunication face au principe de Palo Alto selon lequel " on ne peut pas ne pas communiquer $n$, avant de conclure sur son indispensable conjuration. 
L'apprentissage du code est long, mais en général peu douloureux grâce à une familiarisation, une socialisation de proximité et d'imitation inaugurée dès l'enfance. Mais surtout, cette communication interpersonnelle est présentée comme la réalité communicationnelle la plus riche qu'un homme puisse rencontrer, et c'est cette richesse elle-même qui, parfois, peut engendrer les comportements pathologiques : parce que les codes glissent les uns sur les autres, se confondent, permutent, etc. et engendrent des logiques paradoxales et/ou des double-bind dont il n'est pas toujours, voire rarement, facile de s'échapper.

Cette communication interpersonnelle vaut en quelque sorte comme communication absolue, indépassable (sauf à croire à une communion avec Dieu ou un dieu quelconque), malgré ses contraintes, voire même ses ratés. Modèle fondamentalement anthropo-centré de la communication ou au plus large, biologiquement centré (car les animaux, être vivants communiquent eux aussi). Il en va d'un credo inattaquable sous peine de la plus infamante des condamnations : à savoir celle de déterminisme technique. Or, de l'un à l'autre il y a loin et peut-être, croyons-nous, un espace pour une approche qui manie plus l'hypothèse que l'excommunication.

Bref, notre ambition ici, on l'aura compris, est bien de renvoyer dos à dos le modèle télégraphique et le modèle de l'orchestre. Le premier parce qu'il ne parle que techniquement de la technique, sauf à le trahir d'emblée et lui faire dire ce qu'il ne peut pas dire. Modèle qui vient de l'intérieur de la technique, modèle de technicien, qui n'apporte donc rien sur la technique. Modèle qui n'est pas un discours à propos de la technique, mais une recherche de son optimisation technicienne. Autrement dit, un modèle dont la pertinence reste toute interne à la technique et que nous devons nous empresser de lui rendre. Le second ne convient pas plus : c'est un modèle qui, a priori, n'a rien à voir avec la technique. Il l'exclut même volontiers et la réduit à n'être que de la «vulgaire » technique tout juste bonne pour les techniciens. Modèle non pas extérieur à la technique - position que nous essayons d'adopter - mais bien étranger à celle-ci. Penser la technique à son aune c'est seulement la rapporter à ses problématiques - qui ne sauraient l'envisager en tant que telle - afin d'en montrer - justement - les insuffisances. La technique, ici, a perdu d'avance, elle reste comme boiteuse, amputée, gauche. Elle est en manque de toute cette richesse qui fait la subtilité de la communication interpersonnelle et que même celle-ci a du mal à lui restituer.

Le modèle de l'orchestre part du principe que la communication interpersonnelle, globalement, ça marche, à quelques effets pathologiques près, que l'intervention volontaire du psychothérapeute saura sinon résoudre, du moins déplacer ou relâcher. Or, il convient selon nous, de penser la communication à partir de l'autre bout de la lorgnette : autrement dit, partir du principe que la communication ça ne marche pas, que ça communique mal et que, parfois, ça marche. Au fondement il y a donc l'incommunication ${ }^{2}$ et tous les efforts que les sociétés sont

2. La réflexion autour de la notion d'incommunication est assez récente, mais elle est aussi restée plutôt marginale. Ainsi en 1985, D. Huisman a-t-il intitulé l'un de ses ouvrages "L'incommunication » ( $c f$. Denis Huisman, L'incommunication, Paris, Vrin, 1985). Il souligne que « quand la communication sociale devient pléthorique, abusive et pervertie, elle tend à se transformer en son contraire, c'est-à-dire en incommunication " (p. 85). Cette approche reste dépendante du schéma émetteur- > 
obligées de faire pour la conjurer, la compenser, pour obtenir malgré tout, quelques résultats.

On a tendance à conclure trop facilement $\mathrm{du}$ « on ne peut pas ne pas communiquer » que - donc - ça communique. C'est aller un vite en besogne. Au contraire même, c'est bien parce qu' " on ne peut pas ne pas communiquer " que ça communique trop, de travers, de manière non-maîtrisée, incidente, etc. Bref, la communication interpersonnelle/socialisée, collective, ça communique mal, c'est pourquoi ce n'est sûrement pas à son modèle inversé (celui de l'orchestre) de prétendre constituer un modèle pertinent.

Autrement dit, nous ne partons pas du principe que la "vérité " des TIC se cache inévitablement dans la logique de leurs usages ${ }^{3}$. On ne comprend pas forcément le rôle des TIC de constamment les rapporter à leurs usagers, comme si ceux-ci détenaient le bon modèle de la communication, comme s'ils savaient ce que communiquer veut dire, comme s'ils étaient l'aune indépassable. Penser l'homme comme l'absolu de la communication c'est souscrire à une proposition purement idéologique, alors même que l'on doit penser - et que l'on ne peut penser - qu'à partir de la difficulté qu'il y a-dans toute société - à communiquer et de la nécessité de la conjurer - et pourquoi pas grâce à des TIC (modèle télégraphique), de l'idéologie de la communication... ou même le modèle de l'orchestre (c'est-à-dire l'inscription dans un code, dans une discipline, des normes, des rituels qui cadrent l'action, etc.).

Il nous semble d'emblée important de souligner que cette incommunication ne correspond pas à l'hypothèse d'un état premier et sauvage, elle n'est pas une sorte d'état de nature à la mode du $\operatorname{XviII}^{e} s$, maîtrisé par les procédures diverses

\footnotetext{
- messages-récepteur (EMR), puisque : "l'incommunication des sociétés antérieures à l'apparition des médias résultait surtout d'un manque de puissance des émetteurs oraux et écrits (...). De nos jours l'incommunication provient plutôt, en quelque sorte, de la surpuissance des émetteurs médiatiques (...) » (p. 103-104). En fait cette réflexion sur l'incommunication n'est au fond qu'un détour qui permet de justifier le développement des relations publiques, comme instrument de réduction de l'incommunication (p. 105). Quelques années plus tard, le sociologue R. Boudon propose une « petite sociologie de l'incommunication " ( $f$. Raymond Boudon, "Petite sociologie de l'incommunication $»$, Hermès, $\mathrm{n}^{\circ} 4,1989$ ). Il va s'intéresser, nous dit-il d'abord, aux « difficultés» de la communication, " en d'autres termes, il peut y avoir prolifération des messages et en même temps non-communication dans le cas où ces messages circulent entre des interlocuteurs les percevant à partir d'a priori différents » (p. 53). On le voit, Boudon reste lui aussi tributaire d'un schéma EMR. Il pense l'incommunication comme une "valeur négative » qui progresse de conserve avec l'inculture. C'est en référence à cet article qu'A. Mercier, en 2001, mobilisera à son tour cette notion, mais cette fois en science politique ( $c f$. Mercier, Arnaud, «Amiante, sida, caulerpa taxifolia: l'apport d'une sociologie de l'incommunication à la compréhension des dysfonctionnements des politiques publiques ", in Gerstle, Jacques, Les effets d'information en politique, L'harmattan, 2001). Enfin, tout récemment, D. Wolton vient de proposer de "penser l'incommunication 》 afin de «sauver la communication " dans une perspective à la fois politique et anthropologique ( $f$. Dominique Wolton, Il faut sauver la communication, Paris, Flammarion, 2005). Il soutient ainsi quc « lc cyclc communication-incommunication-cohabitation n'est donc pas l'échec de la communication, mais au contraire la manière de respecter, au mieux, les dimensions normatives de celle-ci » (p. 139).

3. Pour une critique de la notion d'usage, on peut lire : Josiane Jouet, "Retour critique sur la sociologie des usages ", Réseaux, $\mathrm{n}^{\circ} 100$, Hermès, 2000; Serge Proulx, "Usages des TIC : reconsidérer le champ d'étude? ", in Emergences et continuité dans les recherches en infocom, Actes du XII congrès de la SFSIC, 2001 ; Yves Jeanneret et Emmanuël Souchier, "La communication médiatisée est-elle un "usage" ? ", Communication \& Langages, n 132, 2002.
} 
mises en œuvre par la culture (c'est-à-dire par la communication sociale, ses règles, normes et valeurs). Car l'incommunication est toujours là, malgré les procédures et les techniques, parfois à cause d'elles aussi, parce qu'elles n'aboutissent jamais à une parfaite maîtrise, parce qu'elles ne peuvent jamais totalement absorber le réel, qui toujours déborde alors même qu'elles peuvent, en revanche, se faire concurrence, voire s'opposer, se confronter ou se contredire. L'incommunication, nous aurons l'occasion d'y revenir, n'est pas une non-communication, c'est une communication si foisonnante qu'elle met constamment les sociétés au défi de son arraisonnement et de sa maîtrise.

Ce que nous appelons l'incommunication ne fait pas l'objet ici d'une véritable conceptualisation ou modélisation; cette notion vaut d'abord comme question. Il faut la prendre comme un outil heuristique : elle vise à éveiller un autre regard, afin d'ouvrir de nouveaux champs à explorer. C'est pourquoi cette incommunication, nous allons l'aborder à travers des textes qui la mettent en scène : l'Edipe de Sophocle ${ }^{4}$, vaste variation, me semble-t-il, sur ce thème, et Solaris de S. Lem, qui nous confronte directement aux conditions même de possibilité de toute communication lorsque l'on rencontre le radicalement Autre, avant de la confronter au principe singulièrement fort de l'école de Palo Alto selon lequel " on ne peut pas ne pas communiquer».

\section{FoIPE OU L'AVEUgLEMENT COMMUNICATIONNEL}

L'interprétation que Freud nous a offerte d'CEdipe-roi est si puissante qu'elle semble exténuer d'avance toute tentative de dire autre chose sur/d'après ce texte. Peut-on oser relire ce texte autrement que la loi psychanalytique nous y engage désormais? Serait-ce défier quelque part ce père, que de, non pas le contester, mais simplement porter un autre point de vue sur un texte qui, après tout ne lui appartient pas ? Doit-on révérer Freud en père fouettard ? Nous avons la faiblesse de penser que non.

Car on peut voir en CEdipe - et plus généralement dans la tragédie grecque moins les tréfonds d'une psychologie qu'une sociologie, un collectif en relation, tout un travail de la communication ou plutôt de l'incommunication.

Dès le prologue il est question d'abord de Thèbes, de la cité, malade, qui tangue : c'est ce qu'annonce le prêtre comme représentant du collectif. La place d'CEdipe dépend de cette cité, qu'il a sauvée naguère, et qu'il peut sauver encore aujourd'hui, peut-être. Où il est question de politique et de régulation : la peste sévit et le roi, intercesseur, doit satisfaire aux exigences des Dieux pour enrayer la désolation, c'est l'oracle d'Apollon lui-même qui le dit - en témoigne Créon qui revient de Pytho. Le nettoyage sanitaire passe par une prophylaxie morale: que les assassins soient punis, que l'on sache notamment qui a tué Laios auquel Edipe a succédé sur le trône. Le problème posé est d'abord un problème de morale politique, pas forcément de psychologie. Or, le problème est révélé par un messager qui annonce dans quelle voie il convient de s'engager... ce qui ne dit pas, cependant, par où il faut commencer. Ce dont $\mathscr{C}$ dipe se plaint rapidement : « il va de soi que mes recherches tourneraient court si on ne me fournissait aucun 
indice " (p. 110). Intervient alors Tirésias, deuxième messager, qui, aveugle, possède néanmoins le savoir - alors que celui qui voit ne sait rien (p. 115). Tirésias annonce à mots voilés à Edipe que la vérité qu'il cherche n'est pas forcément bonne à savoir - tout au moins pour lui. CEdipe en vient à accuser Créon de trahison et récuse violemment Tirésias. Bref, malgré deux messagers, ou plutôt grâce à deux messagers les choses s'enveniment et deviennent de moins en moins claires... jusqu'à ce que Tirésias annonce tout haut, piqué au vif par CEdipe, de quoi il retourne et de quels malheurs sont frappés les Labdacides. Mais la communication ne passe pas et Edipe ne croit en rien à ces révélations.

Deux messagers se transforment en deux traîtres : plus ça communique, moins ça communique. Autant dire que nous évoluons en pleine incommunication : GEdipe ne sait rien et est aveuglé - avant même d'être aveugle - ; les messagers savent, mais ils ne sont pas crus. Autrement dit, dans un collectif, ça communique mal, et il ne faudra pas moins de tout le reste de la pièce (quatre épisodes sur cinq) pour que la situation globale s'éclaircisse, au prix d'un effroyable assombrissement de celle, locale, individuelle d'Edipe - où, politique, le Roi est celui qui est censé assumer les malheurs du collectif (catharsis) et accepter l'exil après l'avoir proclamé. Commence alors une véritable enquête afin de savoir si l'oracle d'Apollon a été effectivement concrétisé ou non.

Au deuxième épisode, CEdipe accuse de plus belle Créon de félonie (p. 118)... et seule l'intervention de Jocaste - sœur de Créon et femme d'CEdipe - parvient à relâcher la tension. Jocaste, troisième messager raconte alors l'histoire de l'enfant qu'elle a eu de Laios et qui, disparu, ne peut avoir tué son père : CEdipe, vivant, n'étant pas cet enfant n'est donc pas le meurtrier de son père. Jocaste donc, sait. Mais en voulant prouver à CEdipe qu'il ne pcut avoir assassiné son père, elle lui donne quelques indices pour penser le contraire... car Laios a péri à la jonction de deux routes sous les coups d'un inconnu. Or, Edipe a vécu une histoire semblable. Edipe qui prétend et croit sincèrement être né à Corinthe, s'est déjà rendu à Pytho : en fait il sait tout lui aussi (p. 124), mais n'y a jamais cru - car la présence même de son père et de sa mère adoptifs lui laissait penser que l'oracle avait été déjoué. Jocaste sait, $C$ Edipe sait, mais ne veulent conclure a priori au pire. L'information, quand on n'en tire pas les conséquences ne sert à rien, sinon à tromper. Jocaste, troisième messager, bien malgré elle, trahit elle aussi. Au total, on sait, mais ça n'éclaire rien, et lorsque l'on parle on aboutit à l'inverse du résultat escompté. Où l'on ne voit nulle communication, mais beaucoup d'incommunication.

Arrive un quatrième messager, de Corinthe justement. Il apprend à CEdipe que Polybe, son père (adoptif) est mort : mauvaise nouvelle; mais qui en est une bonne tout autant, car si Polybe est mort récemment alors EEdipe n'a pas tué son père, la prophétie a été déjouée (p. 128). Or, le même messager apprend également à CEdipe que Polybe est certes son père, mais son père adoptif : mauvaise nouvelle à nouveau, qui réintroduit la possibilité pour CEdipe d'avoir tué son père biologique. Encore une fois, malgré lui, le messager apporte une nouvelle qui est bonne parce qu'elle est mauvaise (l'innocence d'Édipe est liée aux circonstances de la mort de Polybe), puis devient soit carrément bonne (le vrai père d'Edipe n'est pas forcément mort... Edipe va peut-être éviter de le tuer), 
soit mauvaise parce qu'elle est bonne (car, que le vrai père d'CEdipe soit mort ou non, s'ouvre la possibilité de son meurtre - futur ou passé - par son fils !). Cependant, dans la pièce la première hypothèse de l'alternative n'est même pas envisagée, la dynamique tragique embraye directement sur la seconde : car le messager avoue à Edipe qu'il l'a trouvé dans la montagne, ou plutôt qu'un berger le lui a donné. Ainsi CEdipe-Roi n'est-il peut-être qu'un fils d'esclave. Un esclave de Laios: Jocaste, elle, comprend qu'il s'agit du berger auquel son fils naguère fut confié. Décidément, ces messagers, s'ils font bien leur travail de messager, s'ils apportent de l'information, alors enfoncent-ils encore un peu plus Edipe dans la révélation de son malheur, fermant progressivement les voies possibles de l'espoir. Mais cet apport d'information est lui-même pris dans une dynamique inversée de communication ou du bon naît le mauvais et du mauvais le bon, bref dans laquelle la communication se retourne sur elle-même pour produire des effets inverses de ceux que l'on pouvait attendre; autrement dit, l'information émerge de ces croisements, de ces nouds, elle est le produit incertain de ce processus de communication lui-même incertain - ce que l'on peut donc raisonnablement qualifier d'incommunication.

Le dénouement est aux mains d'un vieillard, d'un simple berger : on l'attend, il arrive. Il résiste. On le menace, alors il avoue que l'enfant était le fils de Laios et qu'il devait l'éliminer à cause d'une prophétie... mais par pitié, il le confia à cet homme. L'enfant et Edipe ne font qu'un, la prophétie a bien été réalisée. Ce cinquième messager qui, dans un premier temps, refuse de communiquer est le porteur de vérité. Mais celle-ci n'est révélée qu'à promettre la mort au vieillard s'il n'obéit pas. Jusqu'au bout, que les raisons soient supposées bonnes ou non, que ce soit volontairement ou non, ça résiste à communiquer. Le fond de l'affaire est toujours celui de l'incommunication, qui est première, qui est aussi à la base même de la dynamique complexe de l'enquête, et qui est encore dernière.

Intervient, enfin, un sixième messager, celui du palais, qui en conclusion, nous révèle la mort de Jocaste et la mutilation d'CEdipe : il est aveugle et enfin, il sait et y croit. Il s'en remet à Créon désormais, requiert de voir ses filles et leur raconte toute l'histoire singulièrement perverse de leur famille : où reviennent sur la scène, à la fois, la famille justement, qui en Grèce est déjà une entité politique, et Créon, qui devient par la force des choses le nouveau souverain. Retour du politique, qui encadre en quelque sorte, en amont et en aval, la dynamique complexe de l'incommunication. Car CEdipe n'a rien voulu, il n'est que le jouet du destin pourrait-on dire, à moins de se demander s'il n'est pas d'abord le jouet de ce processus social fondamental de l'incommunication.

\section{EDIPE : COMMUNICATION OU INCOMMUNICATION ?}

De la communication, d'une certaine manière, il n'en manque pas, mais elle reste toujours inquiète, limitée, entravée, comme si elle ne pouvait pas ne pas s'échapper à elle-même : les prophéties, qui offrent une incomparable information sur l'avenir, tout en semblant le bloquer comme destin, des intermédiaires, des messagers, qui allongent la chaîne et rendent donc d'autant plus facilement possibles et plausibles les bien nommés enchaînements événementiels complexes et incontrôlés. Mais cette communication ascendante si l'on veut, qui va du début 
de l'histoire jusqu'à ce moment du dénouement, est truffée d'informations partielles pour ce qui, localement, semble être la bonne cause : le berger ne dit pas tout ce qu'il sait au Corinthien, qui lui-même et Laios avec, ne diront rien à Edipe de ses origines. Et lorsque l'information parvient à son destinataire, il ne la croit pas forcément : Edipe est prévenu à Pytho, mais n'accorde pas de crédit à la prédiction qui semble infirmée par les faits. La communication est ainsi une sorte d'horizon que l'on n'atteint jamais, parce que l'on ne connaît que le moment local dans lequel on évolue et non pas la carte globale du processus que l'on est en train de mettre en scène au sein d'un collectif. Seuls la prophétie et Tirésias tiennent les tenants et les aboutissants, mais la première fige toute vie en un destin, le graphe des relations est fixé, le programme également, et comme l'on dit, on n'échappe pas à son destin. Le second est condamné à se taire dans l'espoir que les choses, malgré tout, sauront maîtriser la dure loi du destin, se rebeller contre lui. Mais à bien y regarder le plus étrange en l'affaire c'est que, grâce à la fonction totalisatrice de la prophétie, tout le monde sait : Laios, Jocaste, le berger, Edipe, Tirésias, tous ont été amenés à connaître la prophétie. Tous savent, et cela ne sert à rien. Personne ne veut que cela arrive, tous font tout pour l'éviter, mais tous sont pris par les jeux des circonstances et de l'incommunication... qui mène ainsi les routes d'CEdipe et de Laios à se croiser et les deux hommes à se battre. Bref, ça communique, mais toujours mal, ça dérape, ça se retourne, ça se croise, ça se délite, ça s'oublie, ça ne croit pas, etc. Autrement dit, une telle communication, ne vaut-il pas mieux l'appeler une incommunication?

Certes, il y a un orchestre, et personne ne joue sa partition en dehors de l'orchestre - ce que confirme d'ailleurs l'existence d'un destin -, mais cet orchestre, joue-t-il bien - puisqu'au fond il parvient, malgré tout, à réaliser la prophétie - ; ou bien doit-on conclure qu'il joue inévitablement mal - puisqu'il parvient à réaliser, malgré tout, la prophétie ? Tous sont socialisés : Laios, si l'on croit avec lui la prophétie, ne prend-il pas la meilleure des décisions, tout horrible soit-elle ? Le berger obéit, mais l'obéissance à son maître reste modulée par celle qu'il doit à sa conscience. Le Corinthien et Polybe accueillent l'enfant et celui-ci aime ses parents. Jocaste, aime Laios, elle aime CEdipe (comme mari) et elle aime son enfant (Edipe). Sans orchestre, il n'y aurait pas d'accusation de traîtrise, de confiance bafouée ou qui se croit telle... Mais l'orchestre n'explique pas tout, il voile même, en ce sens que les normes morales localement respectées encadrent des comportements qui, globalement mènent à l'immoralité (qui elle-même, dans son jugement de valeur est bien le fruit d'une norme socialement construire et respectée) : comment passe-t-on de l'un à l'autre, il ne l'explique pas, seule l'hypothèse de l'incommunication permet de le comprendre.

Certes, on peut même penser qu'il y a du télégraphe : n'a-t-on pas compté pas moins de six messagers ? Mais ceux-ci n'assurent pas une transmission linéaire de l'information : on le voit le graphe est complexe, enchevêtré même, noué bien souvent. Il ne suffit pas de bien transmettre l'information pour que tout s'éclaire ; et cet éclaircissement engendre une ombre portée des plus considérables. Au contraire de ce que l'on croit souvent, l'objet, ici, circule mieux que l'information, ou plutôt, la bonne transmission de l'objet-CEdipe se paye d'une dégradation stratégique (pour Laios et le berger) ou tactique (ce Corinthien qui ne 
cherche pas à savoir) de l'information : Edipe est conservé en vie, alors même et peut-être parce que l'information s'est évaporée. Edipe vit grâce à cette perte d'information. Celle-ci le mènera cependant à sa propre perte ; et la vérité n'émergera seulement que d'un long processus de reconstruction de l'information par recoupement : une enquête serrée, la piece même de Sophocle. Bref, les fils du télégraphe s'emmêlent et n'expliquent pas grand-chose eux non plus, sauf à considérer la complexité de leur réseau peut-être. Et encore, puisque l'on montre que même la gestion centrale par le truchement de la prophétie n'aboutit au résultat annoncé qu'au prix d'une complexité encore plus grande et localement imprévisible de la gestion de l'information-message. Autrement dit, ce n'est pas la prophétie qui détermine causalement le résultat; celui-ci est le produit des interactions, localement optimisées en fonction de l'information disponible, des différents protagonistes. La prophétie - comme figure bien connue de la justement nommée prophétie auto-réalisatrice - fonctionne comme un attracteur inversé : plus on cherche à s'en éloigner et plus l'on s'en rapproche, plus on veut l'éviter et moins on y échappe. Car tout ce qui est mis en œuvre pour s'en déprendre crée en fait les conditions de son actualisation ${ }^{5}$. Toute information ou le plus souvent tout défaut d'information qui vise explicitement à y échapper y pousse encore un peu plus. N'est-ce pas ce fondement profondément paradoxal de la communication qui la constitue d'abord comme une incommunication, qui engendre le processus même de l'incommunication?

\section{ÇA Marche PARCE QUE ÇA Ne MARChe PAS}

Car on pourrait dire de la communication que « ça marche parce que ça ne marche pas et que ça ne marche pas parce que ça marche ". Entendons que la " communication » est supposée bien fonctionner entre $\mathrm{X}$ et $\mathrm{Y}$, justement parce qu'ils n'ont pas besoin de " communiquer » entre eux. Autrement dit, leurs cadres d'interprétation sont largement superposés et les différences ne sont pas relevées. Ils baignent ainsi dans une " évidence ", mais qui est le fruit d'une construction sociale. C'est pourquoi on peut dire que « ça marche", parce que « ça ne marche pas ", parce qu'il n'y a eu au fond aucune " communication » en tant que processus de négociation du cadre de référence, des règles à appliquer, etc., bref, il n'y a eu aucune métacommunication pour reprendre le vocabulaire de Palo Alto. Lorsque l'on considère qu'il y a un dysfonctionnement de la « communication », que l'on identifie un problème de "communication ", que l'on éprouve réellement le besoin de communiquer, c'est justement parce que ça « communique ", c'est-à-dire que les cadres de $\mathrm{X}$ et de $\mathrm{Y}$ ne s'ajustent pas forcément bien, qu'il y a du jeu, que les différences font saillie, que l'on est donc sorti de l'évidence et qu'il est nécessaire de négocier l'articulation des cadres ou le bricolage d'un cadre commun, voire l'adoption d'un autre cadre, bref qu'il est indispensable de

5. Rappelons que P. Watzlawick a souligné le travail d'un tel mécanisme dans le mythe d'đdipe (cf. Paul Watzlawick, L'invention de la réalité, Paris, Le Seuil, 1988, p. 114); il précise que le philosophe K. Popper avait lui-même discuté de "l'influence de la prédiction sur l'événement prédit [et qu'il avait] appelé cela "l'effet CEdipe", à cause du rôle si important de l'oracle dans le déroulement des événements qui avaient conduit à la réalisation de sa prophétie " (Watzlawick cite Popper, op. cit., p. 114). Je remercie F. Colin de m'avoir remis en mémoire ce passage. 
recourir à une métacommunication. C'est dire que "ça ne marche pas " justement parce que «ça marche ». Toute la tragédie de Sophocle, parce qu'elle est d'abord une enquête, doit être comprise comme un vaste travail de " métacommunication " où se négocient, non sans difficultés, non sans agressivité, non sans incompréhensions, les cadres pertinents susceptibles d'apporter la "vérité ». Une vérité qui, ici, perce, parce que l'on sait dès le début ou presque le fin mot de l'histoire, parce que l'on possède l'aune de la prophétie et que le ressort dramatique tourne justement autour de son avènement, malgré tout. Mais dans la vie il en va tout autrement, car personne ne possède ce méta-point de vue de la vérité, chacun a la sienne. La " métacommunication " n'a rien à voir avec une évidence de la communication, elle ne coule pas de source, elle est un processus long et complexe, qui peut échouer, être suspendu puis reprendre, un processus délicat qui demande des allers-retours, donc du temps, etc. Cette "métacommunication ", nous préférerions l'appeler "méta-cadrage " plutôt, éliminant par là même cette gênante référence à une communication dont nous venons de voir qu'elle disparaît en quelque sorte dans son paradoxe. Méta-cadrage parce qu'il "communique» moins qu'il ne cherche à construire un dispositif minimal de règles d'interprétations communes de la situation susceptible d'extraire les protagonistes de l'incommunication.

Il y a bien du paradoxe dans la communication (mot qu'il convient de lire désormais, ici, toujours avec des guillemets), mais celui-ci n'est en rien une figure de la pathologie : c'est dire qu'il y a toujours du paradoxe dans toute communication. Y voir une pathologie, c'est alors supposer que toute communication est pathologique et doit, donc, être soignée. C'est peut-être faire quelque part le jeu d'une psychologisation généralisée de notre mode de vie, et le jeu d'une certaine idéologie de la communication qui promeut comme sauveurs tous les intermédiaires de la communication. Non, plutôt que de rendre toute communication pathologique, il vaut mieux penser autrement la communication, ou plutôt penser en terme d'incommunication. Autrement dit, il n'y a pas d'abord la communication et parfois quelques dysfonctionnements; il n'y a pas non plus une pathologie intrinsèque de la communication. Il n'y a qu'un processus premier d'incommunication, qu'il convient de prendre comme une forme positive et non pas négative : c'est la condition de toute communication et non pas une pathologie - en ce sens elle ne requiert nullement l'intervention d'une horde de psychothérapeutes. Bref, ça communique mal, et c'est bien pourquoi la société - et il s'agit bien de raisonner en termes sociologiques et non psychologiques - élabore toute une panoplie d'instruments pour que ça communique un peu mieux : mais rien n'est sûr, et l'instrument, de la visée au résultat peut s'avérer plus que trompeur. La société invente donc de l'orchestre et du télégraphe pour assurer, espère-t-elle, une meilleure communication : mais ça ne reste qu'un vœu pieu, un objectif qui est rarement atteint et qui, lorsqu'il le semble localement, peut néanmoins s'inscrire dans une logique globale singulièrement perverse. L'orchestre et le télégraphe nous rassurent encore une fois localement sans pouvoir nous assurer globalement de l'obtention d'un résultat satisfaisant. Sophocle nous montre un modèle ou la dynamique locale rejoint la prophétie globale, et semble vouée à s'inscrire dans la ligne de pente d'un destin, à condition toutefois de croire en la prophétie : mais 


\section{2 ÉPIStÉmOLOGIE}

l'action de la prophétie n'est en rien globale - en ce sens il n'y a pas de destin ni de déterminisme causal -, mais elle-même locale - à vouloir s'en défendre, l'éviter, l'action et l'information en sont déformées. L'orchestre et le télégraphe ne peuvent donc pas constituer des outils d'analyse de la communication mais doivent être appréhendés comme des réponses à la situation d'incommunication première.

\section{SOLARIS OU LE PESSIMISME COMMUNICATIONNEL}

Stanislas Lem a écrit un grand livre - qui est aussi devenu un classique de la science-fiction - sur la difficulté qu'il peut y avoir à communiquer, certes avec les autres humains (car les relations ne sont pas forcément simples entre les protagonistes), mais surtout avec une entité non humaine, une sorte d'océan que l'on est venu à qualifier de vivant et dont on suppose même qu'il est doté d'une forme d'intelligence qui laisse penser qu'il est possible d'entrer en contact avec lui, bref de communiquer ${ }^{6}$. Mais Lem n'a pas écrit une ode à la gloire de la communication, de sa possibilité toujours renouvelée entre les "espèces" les plus diverses, quitte à affronter quelques épreuves qui, surmontées, donneront encore plus de relief à la beauté intrinsèque de la communication. Non, Lem pose à travers et dans son roman la question, beaucoup plus fondamentale, de l'incommunication.

À la base il y a cette dépendance de l'homme à l'égard de «l'outil » qui lui permet de percevoir, de construire - socialement - des représentations, de penser, d'exercer les multiples formes de son intelligence : son cerveau (et après Leroi-Gourhan ajouterions-nous volontiers, son corps). Peut-être toute intelligence ne peut-elle s'exprimer que dans le cadre du support qui l'accueille ? En tout cas tel semble être le cas de l'intelligence humaine, qui d'ailleurs éprouve le plus grand mal à concevoir qu'une intelligence puisse exister autrement, sur un autre mode, sauf à supposer dans un mouvement dialectique vieux comme le monde cette intelligence - qui, pour l'homme confine inévitablement au divin totalement abstraite de la matière, libérée de ses contraintes. Mais supposer n'est pas concevoir : on peut en faire l'hypothèse, sans pouvoir se faire la moindre idée de son modèle opératoire.

À peine le héros de Lem a-t-il débarqué sur la station de Solaris qu'il est en proie à ce qu'il suppose être des hallucinations. Mais justement, tout le problème est là : s'agit-il réellement d'hallucination, d'un produit de son imagination ou bien n'est-il pas en face de quelque chose de réel mais qu'il ne comprend pas, quelque chose qui existerait véritablement indépendamment de lui mais sur un mode qui lui échapperait ? Or, il ne trouvera pas, malgré ses efforts, le critère décisif - "l'experimentus crucis" dit-il (p. 81) - susceptible de l'aider à faire le partage entre ce qui l'habite et ce qu'il côtoie, car « on ne pouvait pas penser autrement qu'avec son cerveau, on ne pouvait se voir de l'extérieur afin de vérifier le juste fonctionnement de ses processus internes (...)» (p. 82).

Où l'on apprend que pour communiquer avec l'Autre, il faut déjà l'avoir identifié, reconnu comme tel - et réciproquement. Et réciproquement, parce qu'il est tout aussi indispensable que l'autre vous identifie à son tour. Ce qui signifie que les deux protagonistes parviennent à ne pas prendre l'Autre pour une

6. Cf. Stanislas Lem, Solaris, Paris, Gallimard, coll. « Folio », 1966. 
hallucination, à concevoir l'Autre comme autre chose qu'une déclinaison du Même, à le détacher du Même : poser la différence qui sépare l'Un de l'Autre, quelque chose qui ressemble à un moi d'un côté et à un toi de l'autre. Car, si l'Autre n'est pas identifié comme tel, s'il reste au fond seulement un sousensemble de ma représentation du monde, totalement asservi à cette représentation, alors le "dialogue " engagé avec lui ne pourra pas être le même que s'il est réellement posé comme Autre. Où plutôt il n'y aura pas de dialogue, puisqu'il participe, que l'on participe du Même; à moins d'accepter un dialogue, mais de toute manière purement interne, au sein du Même: dans les deux cas - même avec ce dialogue internalisé - il n'y a pas communication.

La communication exige donc d'une part une capacité à créer l'autre comme Autrui, mais tout en même temps de le penser quelque part comme Même : il s'agit de le re-connaître, et ce "re " renvoie à ce modele que nous sommes à nous-même (individuellement et collectivement). Il est Autrui, mais équivalent à moi : nous nous devons dès lors mêmes devoirs et mêmes droits.

Or, l'anthropologie nous a montré que nombre de sociétés dites primitives s'intitulaient elles-mêmes "les hommes ", introduisant par là un doute sur la qualité et la qualification des autres, qui donc, ne sont pas forcément, dans un premier temps en tout cas des Autruis. L'histoire nous a enseigné que lorsque les Européens ont débarqué aux Amériques ils se sont - sans être forcément dupes de leur propre question, posée également pour des raisons stratégiques - interrogés sur l'humanité de ces créatures qu'ils rencontraient qui, quelque part ressemblaient à ce qu'ils appelaient homme mais dont ils ne savaient pas si elles étaient ou non dotées d'une âme. La science-fiction nous montre, à son tour, dans une investigation de laboratoire, que le partage entre soi et Autrui n'a rien d'évident, qu'il doit être construit et que cette construction qui ne peut être le produit d'une pure opération logique susceptible de définir le critère de différenciation doit encore beaucoup à l'arbitraire, qui n'est lui-même contenu que par la norme et la convention sociale (où, nous dit encore le romancier, l'amour !). Il n'y a donc pas d'abord et d'emblée la communication - comme son idéologie voudrait nous le faire croire - mais bien cet ensemble complexe de présupposés et de limites même de notre équipement sensoriel et de notre cerveau, socialement mis en forme. Autrement dit, encore une fois, on constate l'existence première de l'incommunication, à laquelle ce que l'on pourrait étiqueter comme communication ne peut constituer qu'une réponse, seconde donc.

Est-ce l'entité, cet "océan » qui cherche à " communiquer " à travers ces "fantômes", ces répliques improbables de personnes connues autrefois - et parfois disparues? Quoi qu'il en soit cet Océan, lui, est bien, là, distinct : mais sa forme de vie pose, avec entêtement, la question de ce qu'il est. Des générations de savants vont s'employer à développer une science solariste, à la recherche de récurrences, de constantes, de lois, de types, etc. Or, "incapables de reconnaître cette vérité, les solariens évitent prudemment toute interprétation du Contact, présenté dans leurs ouvrages comme un aboutissement final, alors que primitivement les esprits lucides le considéraient comme un début, une ouverture, une incursion sur une voie nouvelle, parmi beaucoup d'autres voies possibles " (p. 272-273). Lucides écrit - avec ironie ? - S. Lem, lucides, car ils croyaient en 
effet à la lumière de l'évidence même de la communication; mais l'espoir du contact toujours reporté le transforme en une mystique, une fin "sanctifiée " (p. 273). Où Lem trace en fait les bornes mêmes de la communication : entre la naïveté d'une communication qui se veut toujours un commencement, une ouverture qui reste, malgré ses premiers balbutiements qui ne rajoutent qu'un peu de piquant à sa sauce, somme toute facile, en tout cas, toujours possible et désirable; de l'autre une communication qui n'est jamais actualisée, et dont on pense même qu'elle ne le sera jamais, une communication qui, bientôt, en désespoir de cause, se confond avec l'espérance. Comme si le destin de la communication c'était son écartèlement entre deux positions tout aussi idéologiques, mais qui se rejoignent à la sanctifier, a priori ou dans cet a posteriori paradoxal de son attente. Qu'elle verse dans l'idéologie ou le désespoir sublimé en une mystique, la communication semble ne pas pouvoir échapper à sa radicalisation tout simplement parce qu'elle n'est pas la question, mais seulement une réponse. Une réponse dont la logique de l'extrême est à la fois le symptôme et la mesure de cette énigme de l'incommunication et de la nécessité de la conjurer.

On pourrait qualifier l'attitude de Lem de pessimisme communicationnel. C'est ainsi qu'il en vient à faire dire à son héros qu' « un contact signifie l'échange de certaines connaissances, de certaines notions, ou du moins de certains résultats, de certains états de fait - mais s'il n'y a pas d'échange possible ? Si l'éléphant n'est pas un microbe géant, l'océan n'est pas un cerveau géant. De part et d'autre, évidemment, des tentatives peuvent être entreprises » (p. 230). Or, l'une de ces tentatives - du moins est-ce l'hypothèse avancée - prend la forme d'une femme aimée autrefois et morte depuis, une femme à la « réplique " de laquelle il demande songeur " m'as-tu été envoyée pour me torturer, ou pour adoucir mon existence, ou n'estu qu'un instrument ignorant sa fonction et dont on se sert pour m'examiner comme à travers un microscope ? Se sert-on de toi pour me témoigner de l'amitié, pour me porter un coup insidieux, ou pour se railler de moi ? Peut-être tout cela à la fois, ou peut-être - et c'est plus vraisemblable - s'agit-il de bien autre chose " (p. 230). Communiquer n'est décidément pas simple lorsque l'on ne peut savoir ni quelle forme peut prendre la communication, ni quelle intention on peut concéder à l'autre, alors qu'il est peut-être radicalement impossible de se comprendre. Impossible tout simplement parce que « les notions importées de la Terre n'aident pas à pénétrer les mystères de cette planète " (p. 194). Impossible parce que « du reste que peuvent attendre, que peuvent espérer les hommes d'une "liaison d'information" avec l'océan vivant ? (...) Mais tout cela représente une connaissance intransmissible; transposées en un langage humain quelconque, les valeurs et significations recherchées perdent toute substance - on ne peut les faire passer de l'autre côté de la barrière " (p. 272). Impossible parce que Lem fait au fond sienne cette analyse d'un des savants spécialisés dans l'étude de Solaris: "Grattenstorm [qui] avait conclu qu'il n'était pas question, et ne saurait jamais être question, d'aucun "contact" de l'homme avec une civilisation extrahumaine" (p. 269). Pourtant la Terre a investi des sommes considérables dans l'instrumentation la plus sophistiquée pour apprendre et comprendre : « les explorateurs (...) mettaient en place des appareils d'enregistrement et des caméras automatiques. Les satellites artificiels captaient par télévision le bourgeonnement 


\section{De la communication à l'incommunication?}

des mimoîdes et des logus, communiquant fidèlement des images de maturation et d'anéantissement. Les bibliothèques débordaient, les archives ne cessaient de s'accroître, et le prix à payer pour toute cette documentation fut souvent très onéreux" (p. 194-195)... mais rien n'y fit, le contact ne fut jamais établi ! Ce pessimisme communicationnel constitue une bonne introduction à la reconnaissance de l'incommunication.

Cela dit, l'incommunication ne doit pas forcément être vue comme un déficit qui exigerait impérieusement les réponses de la performance et de l'orthopédie ou de l'antalgie : elle n'a pas à être perçue à travers la lorgnette économico-technicienne de l'efficacité/performance qui ne conçoit comme réponse pertinente que la seule réponse technicienne des TIC - Camus se plaisait à imaginer Sisyphe heureux, pourquoi pas des hommes épargnés par une telle logique ? Elle n'a pas plus à être jaugée à partir de cette vision idyllique de la communication comme cataplasme généralisé aux vertus antalgiques résorbant les blessures infligées par l'être en société : comme on a pu vivre sans ingénieurs ou sans économistes, on peut vivre également sans psychologues et autres techniciens de la PNL.

\section{De Palo Alto à l'incommunication}

L'hypothèse de l'incommunication, au contraire peut-être de ce que l'on pourrait supposer a priori, ne s'oppose pas au " on ne peut pas ne pas communiquer " ${ }^{7} \mathrm{de}$ Palo Alto, elle le relativise. En effet, il est pour le moins impossible de ne pas avoir de comportement et donc en ce sens de ne pas communiquer; il est impossible que notre comportement, quel qu'il soit, ne soit pas interprété par les autres. Mais il ne faudrait pas en conclure pour autant, comme on le fait trop souvent, que ça communique "bien ", ou qu'il en irait d'une sorte d'évidence de la communication, toujours première. D'ailleurs il convient de s'interroger quelque peu sur les mots ; peut-être rencontrera-t-on un abus de langage. Car, s'il est vrai qu'il n'existe pas de négatif du comportement, dans le sens de la possibilité d'avoir un noncomportement, cela signifie que tout comportement en société ne peut pas ne pas être interprété par les autres; de là à conclure de ces interprétations croisées qu'il y aurait communication, c'est, nous semble-t-il aller un peu vite en besogne. On ne peut pas ne pas interpréter et ne pas être interprété, certes, mais chaque interprétation et leurs enchevêtrements produisent-ils pour autant de la communication ?

Pourquoi conserver un mot, " communication ", qui possède une indubitable connotation positive, pour désigner un processus relationnel porteur d'effets de réciprocité et de miroir ? À consulter le dictionnaire Le Robert on constate que le modèle de la communication télégraphique domine l'ensemble des définitions ${ }^{8}$.

7. Cf. Paul Watzlawick, Janet Beavin et Don Jackson, Une logique de la communication, Paris, Le Seuil, 1972, p. 46.

8. En effet, que ce soit "établir une relation", « communiquer quelque chose à quelqu'un ", ou la " chose que l'on communique ", les "moyens techniques", voire "le passage d'un lieu à un autre ", il n'en va d'aucune connotation négative. Celle-ci n'affecte que l'interruption de la communication. Le verbe communiquer relève également d'une connotation positive : que ce soit "faire connaître ", " faire partager », « rendre commun, transmettre » ou " se mettre en relation ", voire " être en relation par un passage ». Et même l'usage pronominal ancien et rare nous dit-on, " se communiquer » dans le sens de se confier, attesté par une citation de Montesquieu, ne renvoie à aucune stigmatisation. 


\section{$16 \mid$ ÉPISTÉMOLOGIE}

Même la référence aux sciences sociales renvoie à la sociologie des mass-médias (présente dans l'édition de 1983 elle est absente de celle de 1990). Et ce modèle est positif parce qu'il signifie le passage; sauf à " couper, fermer ou rompre les communications ", opérations toujours marquées du sceau du négatif. Or, le modèle de l'orchestre vise justement à mettre en évidence, non un passage, mais le jeu d'une herméneutique interactionnelle dynamique, le miroitement des interprétations réciproques, la richesse des effets de système, l'entrelacement des feed-backs... n'est-il pas dès lors pour le moins raisonnable de douter de l'adéquation du mot à la chose?

Autrement dit, lorsque l'on croit parler de communication, on recouvre d'un mot très vague une situation complexe, on la rationalise, on essaye déjà de la maîtriser plus que de la comprendre. Penser "communication " c'est déjà charrier un nombre considérable de présupposés dont le moindre n'est pas de laisser entendre que, bien évidemment, ça communique, que la normalité, c'est justement la communication. Donc que, lorsque quelque chose bloque, lorsque manifestement on ne s'entend pas on est justifié à qualifier le processus de "dysfonctionnement " (où si l'on est dans le modèle du télégraphe, de bruit), de poser un diagnostic de pathologie de la communication. Sans provocation, nous dirions volontiers que c'est la pathologie, c'est-à-dire ce qui est traditionnellement étiqueté comme tel, qui, en matière de "communication " est la règle, la normalité : c'est pourquoi nous concluons à l'incommunication ; c'est pourquoi cette incommunication doit être considérée comme le fondement à partir duquel il devient possible de penser en toute sérénité la " communication ».

Oui, l'homme est un animal sémiotique qui ne peut pas ne pas interpréter le monde et les comportements des hommes eux-mêmes. Mais ces interprétations, dont les cadres de base sont des produits de la société, sont convoquées de manière brusque, inopinée, bricolées à la hâte pour servir ici et maintenant, dans le feu de l'action à se repérer, à s'orienter et à agir, non pas en connaissance de cause, mais en fonction des données partielles, tronquées, floues, qu'elles nous offrent. Bref, ça interprète tous azimuts, de tous côtés, mais toujours dans une large incertitude, sous contraintes, avec une "information " légère, discontinue, rarement cohérente à laquelle les cadres offrent, malgré leurs propres faiblesses, des solutions de continuité toujours incertaines, parfois improbables. Conclure d'un tel processus que ça communique ce n'est plus seulement accepter une définition faible de la communication, c'est tendre vers son degré zéro. Il nous semble plus simple - et plus judicieux - dès lors de penser en terme d'incommunication. C'est dire que la communication, si elle existe, ne peut venir qu'en seconde vague, qu'elle ne peut émerger que d'un travail long et difficile. Elle n'est pas donnée, elle est à construire et il convient de s'interroger justement sur le processus social et les moyens de cette construction. C'est dire peut-être aussi, en poussant le raisonnement à sà limite, qu'elle n'est qu'un horizon qu'il n'est pas possible d'atteindre parce que tout effort en ce sens joue, pratiquement, de manière totalement contre-productive.

L'hypothèse de l'incommunication, si elle relativise le modèle de l'orchestre - en montrant que la communication est une question là on la considère comme une évidence, à sa pathologie près, en vient plus encore à disqualifier le modèle 
télégraphique. Car ce dernier voit en la communication un processus totalement évident, à cette manière de pathologie près appelée "bruit ", puisque le classique schéma de la communication est censé permettre d'aborder n'importe quelle situation de communication : ce modèle est typiquement un "presse-purée " qui produit de la communication (ou son dysfonctionnement) à tour de bras. Modélisation ajustée à une réalité technique interne (celle du téléphone notamment) et qui devient véritablement simpliste lorsque l'on prétend l'utiliser pour appréhender des situations sociales de communication et même lorsque l'on croit pouvoir y avoir recours pour modéliser les TIC en situation sociale (comme s'il pouvait en être autrement d'ailleurs).

$\grave{A}$ travers cette loupe on voit de la communication (et ses dysfonctionnements) partout, et même là où il n'y en a peut-être pas : autrement dit, on ne pose jamais la question de savoir ce que communiquer veut dire. Soit, ça communique (car n'importe quelle situation peut être regardée au travers d'un tel schéma comme "communicationnelle»), soit ça dysfonctionne, parce qu'il y a du bruit, à identifier, puis à éradiquer pour revenir à la normalité, à savoir que " ça communique ». La modélisation n'est pas une étape dans un travail global de problématisation, elle n'est pas un outil au service de la construction de cette problématisation, elle s'y substitue. Comme souvent avec les modèles d'ingénieur dont l'objectif est d'intervenir de manière la plus efficace possible sur le réel, le modèle est là pour fournir cette aune de l'efficacité que le réel ne possède pas forcément et que l'ingénieur manie avec dextérité : grand chasseur de dysfonctionnements, il les détecte grâce à son modèle et les réduit, voire les supprime quitte à plier pour ce faire la réalité à sa volonté - car seule l'efficacité prime.

Avec un tel schéma toute incommunication ne peut être assimilée qu'à du bruit qu'il convient donc d'évacuer et non comme fondement même de toute possibilité de communication. Il ne s'agit toutefois pas d'interpréter l'incommunication comme une sorte de modèle de l'ordre par le bruit : car je ne dis pas que c'est l'incommunication qui produit la communication, je ne dis pas que la communication sort de l'incommunication, mais que les sociétés élaborent des systèmes de normes et de règles, de l'idéologie aussi, voire des techniques, afin de construire quelque chose qu'elles étiquettent comme étant de la «communication ", ou quelque chose qui fonctionne comme ce que l'on pourrait alors appeler "communication". L'incommunication est commune à toutes les sociétés, ce que nous baptisons " communication » sans savoir forcément exactement de quoi il s'agit, ne l'est pas; ce qu'elles partagent toutes cependant ce sont ces efforts de mise en forme afin d'essayer de sortir de l'incommunication, efforts dont il reste à dresser la typologie des modalités et des moyens (dont nous effleurons seulement l'esquisse dans le cadre de cet article). En ce sens « orchestre » et "télégraphe » ne sont pas des modèles, mais seulement deux classes possibles au sein de cette typologie.

L'incommunication doit être comprise comme un problème, une question à laquelle les sociétés doivent trouver une réponse. C'est-à-dire qu'elles vont construire des réponses fort diverses (dans leurs formes, leurs supports, etc.) mais qui, toutes vont participer à conjurer l'incommunication. Conjurer ce n'est pas supprimer, c'est faire face en se protégeant ; conjurer c'est écarter, dissiper, 


\section{$\mathbf{1 8} \mid$ épistémologie}

détourner par un moyen quelconque (une prière, un rite, un objet) quelque chose qui met en cause le collectif, sa cohésion, la possibilité même de sa cohésion. Nous avançons l'hypothèse que le meilleur moyen de conjurer la question de l'incommunication, tout au moins dans notre société, c'est d'en rendre la réponse " évidente ", c'est-à-dire de la mettre en scène sous l'angle de l'évidence, de lui faire adopter la posture de l'évidence. Autrement dit, on conjure d'autant mieux que l'on n'a à justifier et/ou à expliquer ni la réponse, ni la question. C'est exactement ce que notre société fait avec ses TIC: le discours qui les présente comme irrécusablement indispensables les inscrit pleinement sous l'orbe de l'évidence et leur permet de ne pas avoir à se justifier, c'est cela que j'appelle l'impensé ${ }^{9}-$ mode sur lequel existe l'informatique dans notre société. Les présenter ainsi autorise donc notre société à proposer les TIC comme solution aux multiples situations sociales identifiées comme relevant d'une pathologie de la communication : on invente ainsi à la fois la pathologie et le remède qui va avec. Cette position qui relève de l'idéologie de la communication a justement pour fonction de conjurer dans nos sociétés contemporaines, l'incommunication première.

Le terme "communication ", comme catégorie d'analyse, pose plus de problèmes qu'il n'en résout, dans la mesure même où il est habité par une connotation positive. Si les SIC acceptent de le conserver, elles sont obligées de glisser sous cette étiquette des processus complexes qui entrent en totale contradiction avec cette connotation positive. Cela ne pose-t-il pas un véritable problème de cohérence ? Si les SIC se définissent par l'adoption d'une « posture communicationnelle ", comment, dès lors, peuvent-elles critiquer ce qui les constitue, sans quelque part se renier elles-mêmes? Cela ne pose-t-il pas un réel problème logique, alors même que la connotation intrinsèquement positive de la notion de communication renforce considérablement cette résistance à la critique ? Il est donc du devoir des SIC de ne pas croire en la communication ${ }^{10}$ : elles sont là pour comprendre et tenter d'expliquer; ne pas croire signifie qu'il faut assumer jusqu'au bout ce que cela implique : renier la notion même de communication comme point de départ pertinent au profit, pourquoi pas, de la notion d'incommunication - et de sa nécessaire conjuration.

\section{PASCAL ROBERT}

9. Cf. Pascal Robert, "Confiance, technique et justification, le rôle du macro-techno-discours informatique dans l'établissement d'un "climat de confiance" ", Quaderni, n 46, Hiver 2001-2002, Paris, p. 53-66. Pascal Robert, L'impensé informatique, Thèse sous la direction de P. Breton, Université Paris-I Panthéon-Sorbonne, 1994. Voir aussi, Pascal Robert, Le modèle CRITIC, HDR, sous la direction d'Y. Jeanneret, Université Paris-IV Sorbonne, 2004.

10. Comprenons : il s'agit bien de ne pas croire en la communication comme concept, comme notion théorique ; ce qui ne signifie pas forcément qu'il ne faille pas y croire en tant qu'homme et/ou citoyen - plan sur lequel se situe, me semble-t-il, D. Wolton lorsqu'il milite en faveur de la communication (cf. Wolton, op. cit). 